\title{
Power and Responsibility
}

An Equity, Diversity, and Inclusion (EDI) Community Paper to the
2020 Planetary Science and Astrobiology Decadal Survey 2023-2032

\author{
Lead Co-Authors \\ Daniella Scalice, NASA Ames Research Center (daniella.m.scalice@nasa.gov) \\ Melissa Kirven Brooks, PhD, NASA Ames Research Center \\ Aaron Gronstal, PhD, NASA Ames Research Center
}

Co-Authors:

Moses Milazzo, PhD, Other Orb, LLC

Lauren Seyler, PhD, Stockton University

Jamie Foster, PhD, University of Florida

Nathalie Cabrol, PhD, NASA ARC/SETI Institute

Loren Williams, PhD, Georgia Institute of Technology

Darlene S. S. Lim, PhD, NASA Ames Research Center

Jackie Goordial, PhD, University of Guelph, Canada

Endorsements:

Timothy Holt, University of Southern Queensland

Kat Gardner-Vandy, PhD, Oklahoma State University

Devanshu Jha, MVJ College of Engineering, Bangalore, India

Abel Mendez, PhD, University of Puerto Rico at Arecibo

Shawn Domagal-Goldman, PhD, NASA GSFC

Nicole Whelley, University of Maryland, College Park/NASA GSFC

Julie Rathbun, PhD, Planetary Science Institute

Ayodele Adekunle Faiyetole, PhD, Founder at EarthSpace, and Senior Lecturer at the Federal University

of Technology Akure, Nigeria

Kevin Hand, PhD, Jet Propulsion Laboratory

Kunio M. Sayanagi, PhD, Hampton University

Rory Barnes, PhD, University of Washington

Benita Bell, PhD, Morgan State University/NASA GSFC

Tori Hoehler, PhD, NASA ARC

Penelope Boston, PhD, NASA ARC

Nicolle Zellner, PhD, Albion College 
Summary of Recommendations:

- Agencies enact a new, stepped-implementation strategy in their solicitation, review, selection, and award processes.

- Next issuance of solicitations require proposals to include the proposing institution's policies and practices in Diversity, Equity, Inclusion, and Access (DEIA), and related information/data describing success or failure on these fronts.

- Subsequent issuances require proposals to include this same information, and will supply criteria (developed through external expertise) against which it will be evaluated.

- Special review panels are convened to evaluate this information separately from those that review the proposed science and technology.

- As part of the awarding process, negotiations can take place between agency and institution toward procurement of the proposed work and any needed policy remediation; proposals can also be rejected on the basis of low DEIA scores.

- Through this process, agencies gain knowledge and wisdom to evolve their own DEIA policies and practices toward the development of a safe, diverse, anti-racist workplace.

\section{Summary of Impact:}

By 2032, it is imagined that this new process will ensure the science and technology that agencies procure and support are able to develop in institutional environments reflective of excellence in anti-racist policy and practice. Agencies will no longer be complicit in the perpetuation of the policies and practices of any institution to which they transfer funds that actively or passively allow racism, sexism, homophobia, transphobia, ableism, classism, colonialism, and other insidious thought processes, value systems, and systemic or individual behaviors to persist.

With the full participation going forward of people of all colors, genders, abilities, walks of life, and ways of loving and praying in a working environment where they can bring their whole selves and be free from harm, agency-funded achievements will authentically represent the humanity that planetary exploration aspires to serve.

Introduction:

Creating anti-racist culture must begin by examining power dynamics. Each party must acknowledge its position with respect to power in any given relationship. Awareness and understanding can transform unhealthy, imbalanced power dynamics. Effective relational and cultural communication will serve as a bridge to create and strengthen an anti-racist environment. The work continues by making positive changes to share power through co-creativity.

NASA has power. NSF and other agencies have power. They are in a dominant position of power in almost every relationship in which they find themselves. They also have the great privilege of managing and spending hard-earned taxpayer money. With this privilege comes a tremendous responsibility to ensure no person is harmed by their actions. To advance anti-racist policy and practice within their own structures as well as those of their stakeholder and partner institutions, agencies should appropriately wield their power and influence to affect positive and lasting change.

Background:

One major way through which agencies express their power is via the solicitation/proposal/review/award process. Solicitations are potent documents. They codify values and communicate priorities and desired 
outcomes. They guide the scientific and technological development of entire fields of study, and focus the community toward shared goals and objectives.

Solicitations are flexible, lithe, and can easily morph to reflect emerging priorities. For example, in the late 1990s, NASA's Science Mission Directorate (SMD) implemented a new policy to reflect its commitment to STEM education and ensured the policy was reflected in its solicitations. The policy required that education be incorporated into mission plans and Research and Analysis (R\&A) proposals. The development of a spaceflight mission was not complete, nor could a scientist propose to take money from SMD for research unless an education program was included. That led to a robust Education and Public Outreach community embedded within SMD and its stakeholder and partner institutions that persisted until very recently, with diverse external partnerships, cross-mission collaborations, and far reaching networks. The same can be true for efforts in Diversity, Equity, Inclusion, and Accessibility (DEIA). Long-term, sustainable commitment to new policies and practices will be required to monitor change and ensure stability.

There is certainly much more to it, but to first order, the awarding of funds to an institution by an agency for a set of deliverables defined in a proposal in response to a solicitation comprises a transaction. The two parties at the nexus of that transaction, primarily, are the agency and the proposing institution, not necessarily the agency and the researcher directly. Indeed, lead personnel at the institution who carry out the proposed work can and do change, most often with the agency's blessing. It's also possible for a researcher to negotiate with the agency to take their awards with them if they change institutions, but even in these cases, the primary seat of the relationship becomes the new institution.

In making an award, agencies are procuring a set of activities and deliverables by a group of researchers embedded in one or more institutions. We must acknowledge that in so doing, agencies are also-via the payment of indirect costs and overhead fees-directly funding, accepting, and implicitly condoning all the policies and practices of the institution. To be sure, most of these are benign, and entail ensuring personnel have what they need in terms of facilities, training, administrative support, etc. They can also entail the requirement that the institution abides by laws and regulations as defined by Congress and interpreted by various regulating entities. Yet institutional policies and practices can, actively or passively, allow racism, sexism, homophobia, transphobia, ableism, classism, colonialism, and other insidious thought processes, value systems, and systemic or individual behaviors to persist. Without deeper analysis, agencies can find themselves complicit in their perpetuation.

Agencies are not unaware of or passive about the policies and practices of those with whom they do business and maintain relationships, even in unfunded arrangements. For example, NASA does not engage in collaborations with certain countries. NASA employees and awardees are presently in a Congressionally directed situation where they are unable to collaborate unilaterally with colleagues in China because of distrust and fear of losing intellectual property resulting from those collaborations. Congress mandates that many ideas, analyses, and designs be kept out of the hands of 'foreigners' through the International Traffic in Arms Regulations (ITAR), and NASA keeps a tight lid on possible violations. There are also numerous countries to which travel is restricted for federal employees by the US State Department. In these cases, scrutiny is applied to protect US citizens and prevent affiliation with nations whose policies and practices are deemed unsafe, untrustworthy, and of low integrity. The same scrutiny must be applied to all agency relationships, transactional or otherwise, especially where the prevention of harm from discrimination and systemic oppression is concerned.

NASA's mission and values touch every state in the nation, as the agency currently awards more than \$1 billion of taxpayer money annually in grants to some 750 science, technology, engineering, and mathematics (STEM) programs at various institutions, including universities and colleges, science centers, museums, research institutions, and other organizations. In 2018, NASA's Administrator issued a policy 
statement reaffirming the agency's commitment to equal opportunity among the many institutions nationwide that receive NASA grant funding or that participate in agency-conducted programs. Under federal civil rights laws, recipient institutions of agency grants, awards, and cooperative agreements must ensure equal opportunity to their program beneficiaries, including STEM faculty, staff, students, and visitors to public science museums and centers.

Despite this, many institutions are not achieving excellence in equal opportunity, diversity, equity, inclusion, or accessibility. Thankfully, many institutions are rising to the occasion of this historic moment in time and the opportunity it presents to engage in truth, reconciliation, and change-making. Many agencies are doing the same in different ways, one of the most high profile of which was NASA's announcement in July, 2020 of a fifth core value in its mission statement: Inclusion.

As part of this commitment, NASA and other agencies must use their power, in the best of ways, to examine how those with whom they do business espouse and implement policies and practices to advance DEIA ideals. To do otherwise risks agencies remaining cogs in the wheel of structural racism, and finding themselves at odds with their missions of cultivating anti-racist culture and a diverse and inclusive STEM workforce.

Recommendations:

\section{A Stepped-Implementation Strategy for Solicitations}

A new process can be imagined wherein solicitations are a key modality to ensure the science and technology the agencies procure and support is able to develop in an institutional environment reflective of excellence in anti-racist policy and practice. Through a stepped-implementation strategy guided by specific goals, agencies can maintain the mission-critical research they support and promote DEIA excellence at the institutions that carry it out.

In the next release of each solicitation they issue, agencies can require proposers to list and describe all institutional policies and practices pertaining to DEIA, and provide data describing success or failure on these fronts. Asking for this information signals to researchers and institutions that the agency is committed to encouraging effective DEIA practices and is paying attention to what's happening at the institutions it would fund. For these first issuances, however, the recommendation is that only the proposed scientific work be evaluated.

In the subsequent release of each solicitation, agencies can require these same pieces of information about the DEIA policies and practices of the institution, and provide criteria against which they will be evaluated, citing indicators of alignment in a rubric from novice to expert (vs. poor to excellent). Agencies can describe in the solicitation how these DEIA scores will factor into the overall assessment of the proposal, and how much weight they will carry in relation to the evaluation of the scientific and technical merit of the proposed effort. Precedent exists for such gradual change to solicitations in NASA/SMD's Data Management Plan (DMP) requirements, which began as suggested content and are now required content, including requiring a budget for the DMP.

Separate review panels can then be convened, one holding specific expertise in the science and technology being proposed and the other in institutional DEIA policy and practice, each tasked to evaluate the proposal's contents in their areas of expertise against carefully developed (via external expertise), stringent criteria put forward by the agency. Program officers and selecting officials, having calculated the scores for each proposal, will then organize proposals into what is selectable and what is not, and why. Thus it will be possible for a proposal to have described desirable, fundable scientific work which would be conducted in a DEIA-poor institution, and for that reason alone, render it unselectable. 
This process opens up spaces for positive, healthy negotiations. Agencies can exert their power, strength, and leadership in this dynamic to draw out racist policies and practices and offer support to the institution toward DEIA evolution and active solutions. Selections or funds can potentially be withheld until change toward anti-racist culture is made. Conditions could be asserted under which the researchers can be enabled to do their work while tracking proposed changes to DEIA policies and practices at the institutional level. Separate pots of funds can be made available to which researchers can propose to enable DEIA capacity building at the institutions of either selected and/or unselectable proposals. Proposals that fail to meet the standards of DEIA excellence can be rejected and agencies can take their business elsewhere to places where they can be assured they're not complicit in structural racism. In any case, this process creates an urgency for the researcher and the institution to engage in self-examination around DEIA, and for the researchers in particular to realize their stake in and take action around how their institution conducts itself. The objective is not to punish, but to identify areas of growth and call everyone to the table to make progress.

As in the case with the DMPs, as these processes unfold, agencies may consider hosting workshops for proposing researchers and institutions to provide insight on how they expect these changes to manifest, highlighting the spirit in which they are being undertaken. This would provide an opportunity for iteration, positive feedback loops, and co-creativity to flourish.

On the practical level, to support these changes in how they conduct business and fund science, agencies must engage external experts to develop the criteria against which to determine DEIA excellence or lack thereof in the institutions to which taxpayer money is being provided. External expertise will also be required to monitor DEIA compliance, especially where new institutional policies and practices are enacted. These criteria can be co-developed by and should be shared across agencies and other organizations.

\section{Dual-Anonymous Reviews}

It is noted that NASA and others are experimenting with a dual-anonymous peer review process for research solicitations in a number of programs. This review system is a step forward in eliminating bias from the process of selecting proposed research. Proposers will remain unaware of the identity of peers who review their proposals, and the reviewers themselves will have no knowledge of either the proposing teams or the proposing institutions during the evaluation process.

As the implementation of dual-anonymous review expands, it will help to address bias when analyzing the scientific and technical merit of research proposals. The same is true when evaluating the DEIA policies and practices of proposing institutions. A dual-anonymous system ensures that the perceived reputation of an institution does not replace or outweigh the actual practices of that institution in building and maintaining an anti-racist culture. A proposing institution will be assessed anonymously each time a proposal is put forward. This not only ensures that an institution has effective practices and programs in place at the time a proposal is submitted, but also that the proposing team is aware of, and engaged with, current practices and programs at their institution.

\section{DEIA Evolution within the Agencies Themselves}

Adjacent and parallel to this entire, multi-year process, there needs to be an internal process unfolding within the agencies. A journey of truth to examine, name, and take responsibility for their role in perpetuating structural racism. A journey to understand how the hubris and entitlement of colonialism and cultural hegemony have resulted in the cognitive imperialism of the scientific enterprise writ large, and how this has affected who gets to participate. We recommend agencies seek external expertise for this, and prepare to make a long term commitment, investing on behalf of their current and future workforce 
to support their individual and collective journeys toward awareness, healing, diversification, and DEIA evolution.

The external expertise toward DEIA criteria development, monitoring, and compliance that agencies will apply for its awardees can be utilized and applied within their own walls. To support this, agencies may find it useful to collect and analyze data in a baselining effort to understand their own current DEIA

profile. Those data can be used to identify strengths and weaknesses, and set measurable DEIA goals and objectives.

However the internal and external processes described above unfold, agencies have an opportunity to cocreate anti-racist culture both for themselves and for the communities of which they are integral part and in which they hold power. They have the opportunity and the responsibility to ensure there is never again a day where the scientific and technical achievements they facilitate are marred by the ugliness of racism. Without the full participation going forward of people of all colors, genders, abilities, walks of life, and ways of loving and praying in a working environment where they can bring their whole selves and be free from harm, those achievements are incomplete, inconsequential, and invalid, and fail to authentically represent the humanity that planetary exploration aspires to serve. 